\title{
Community manager, un perfil hiperespecializado del comunicador social
}

Community manager, a hyperspecialized profile of the social communicator

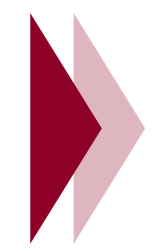

Verónica Martínez Guzmán

Facultad de Ciencias Sociales y Humanas, Uniminuto, Bogotá, Colombia.

Correo electrónico: oldmaguz@hotmail.com

Jorge Iván Cabrera Durán

Facultad de Ciencias Sociales y Humanas, Uniminuto, Bogotá, Colombia.

Correo electrónico: jcabrera1@uniminuto.edu.co 


\section{Resumen}

El objetivo general de este artículo es analizar el concepto del community manager desde una perspectiva integral. Su importancia radica en repensar el papel de comunity manager desde su formación académica; aportar significativa y sustancialmente a la formación profesional y académica del nuevo comunicador social; y crear un perfil eficiente para el sector productivo desde la ocupación práctica a nivel empresarial del comunity manager a partir de la situación problémica que describe ¿Qué oferta académica existe en Bogotá - Colombia para la formación de comunity manager desde los programas de comunicación social? Metodológicamente se sigue un esquema cualitativo - descriptivo bajo la técnica de análisis de contenido. El resultado propuesto consiste en el diseño de una propuesta formativa en comunity manager como experto ocupacional en el campo de la comunicación social.

Palabras clave: comunicación, community manager, academia, IES, currículo.

\section{Abstract}

The general objective of this article is to analyze the concept of the community manager from an integral perspective. Its importance lies in rethinking the role of community manager since its academic training; contribute significantly and substantially to the professional and academic training of the new social communicator; and create an efficient profile for the productive sector from the practical occupation at the business level of the commu- nity manager from the problem situation that describes What academic offer exists in Bogotá - Colombia for the formation of community manager from the social communication programs? Methodologically a qualitative - descriptive scheme is followed under the content analysis technique. The proposed result is the design of a training proposal in community manager as an occupational expert in the field of social communication.

Keywords: communication, community manager, academy, HEI, curriculum.

\section{Introducción}

Los objetivos específicos de este trabajo giran en torno al análisis de la formación en comunicación social de pregrado ofertada por IES privadas en Bogotá - Colombia, con el ánimo de establecer si existe o no un perfil hiperespecializado de la profesión en el campo de la gestión de contenidos web. El problema fundamental que motiva la generación de esta investigación es ¿Qué oferta académica existe en Bogotá para la formación de comunity managers? Como preguntas derivadas se establecen las siguientes ¿Cómo se estructuran curricular y organizativamente los programas de formación existentes? ¿Qué contenidos esenciales contienen? y ¿Qué contenidos están ausentes o insuficientemente tratados y deberían incorporarse en futuras acciones formativas o ediciones existentes? El trabajo realizado cobra importancia al forjarse como un proceso investigativo, nacido de la necesidad de la academia por desarrollar las funciones sustantivas, que le dan importancia y la convierte en epicentro del avance del conocimiento para la 
sociedad a la cual responde y el desarrollo del sector productivo, al propender por la formación de profesionales competentes.

\section{Desarrollo}

El comunity manager - $\mathrm{CM}$ es un comunicador innato que ve al "público como una masa con particularidades que la caracterizan, estudiando el efecto de las publicaciones a nivel individual, grupal, social e institucional, yendo más allá de lo general y enfocándose un poco más en las características particulares de los grupos sociales" (Durkheim, 1895, p.11). El tema en general es nuevo, aún hay muchas personas y empresas que desconocen qué es esto de la social media, cómo funciona y que beneficios reporta.

Según Rojas (2011) citado por Ortega (2016) "la figura del comunity manager puede ofrecer a la entidad que cuenta con ella la cobertura de necesidades más específicas, por ejemplo, reducir sensiblemente el tiempo dedicado a la atención telefónica, ofreciendo a un sector de clientes esta atención a través de las redes sociales que este profesional administra". Las primeras personas que realizaban lo que hoy ya está establecido como "actividades de comunity manager" (Ortega, 2016, p.199), eran algunos de los empleados de las mismas compañías, personas con habilidades multifacéticas que, de formas totalmente ortodoxas, intuían las necesidades del cliente y creían que trabajaban para satisfacer las mismas.

La perfilación, diseño y formulación de todo lo relacionado con el comunity manager, es de reciente data, tanto así que la oferta aca- démica presentada por las universidades en este campo es incipiente, pero pertinente con relación a su demanda empresarial, en este sentido, la Universidad a Distancia de Madrid, España UDIMA, es pionera en esta materia, con el grado de Periodismo Digital y Redes Sociales, que oferta desde 2009.

No obstante, ante la ausencia de una oferta académica de pregrados para el perfil específico del comunity manager, objetivado como perfil hiperespecializado del comunicador social, las universidades en línea, se han convertido en fórmula principal de certificación académica de competencias asociadas al perfil ocupacional del CM, a través de diplomados o cursos virtuales cortos, como los propuestos por el Instituto Argentino de Computación IAC, el Centro de Estudios e Investigación en Comunicación Social, y el diplomado en marketing digital y community management de la Universidad de Ciencias Empresariales y Sociales - UCES en Argentina y algunos grados técnicos como el 'técnico en social mMedia y comunity manager del Centro de Formación en Tecnologías de Información y Comunicación en Costa Rica. Aunque, en la actualidad en México se oferta un programa denominado licenciatura en mercadotecnia digital y comunity manager en el Instituto de Estudios Universitarios - IEU que es $100 \%$ en línea.

Otra opción para certificar competencias en esta área, son los postgrados (especializaciones, maestrías o doctorados), entre los que cabe mencionar la especialización en comunity manager en la Universidad Abierta de Cataluña, el Máster en community management: empresa 2.0 y redes sociales del Instituto de Educación Superior de Brasilia - IESB, y el doctorado en marketing social y redes sociales 
de la Escuela de Estudios Humanos y Sociales de la Atlantic International University - AIU.

Según Fraile (2018), en Colombia, para ejercer este oficio, generalmente, se pide tener un título profesional en áreas como comunicaciones, periodismo, mercadeo, publicidad, relaciones públicas, entre otras. Este autor sostiene, que de acuerdo con las cifras de comunity manager que arroja Linkedin, existen 7.140 personas que desempeñan esta actividad en el país, y que, si se cambia al término de "social media", Colombia cuenta con 65.382 personas que trabajan en este campo. Indica Fraile (2018) que en Colombia hay casos en los que incluso una misma persona administra las redes sociales de hasta ocho marcas y recibe un sueldo por debajo del mínimo (\$781.242), sin embargo, según experiencia y tipo de organización, el salario de este profesional oscila entre los \$ 600.000 y 5.000 .000 de pesos.

En Colombia el comunity manager se asume como perfil ocupacional multidisciplinar, "necesita saber de marketing, comunicación, operaciones y finanzas, pero sobre todo debe tener unas excepcionales habilidades relacionales y de negociación, y una clara orientación al cliente y a sus resultados [...] aunque no todos los días escuchemos la palabra comunity manager; podemos ver como en el país es una figura exponencial" (Haro, 2010, p.71), a través de eventos como el Community Manager Summit Javeriana o Digital Managers Summit Javeriana; ofertas laborales publicadas a diario en portales de empleo como Computrabajo o LinkedIn Jobs; y páginas de Facebook como "Community Managers en Colombia" que cuenta con 24.679 miembros y más de 300 publicaciones mensuales de ofertas laborales para este perfil, la mayor parte de ellas ubicadas geográficamente en Bogotá.
En consecuencia, se seleccionaron cinco programas académicos en comunicación social en la ciudad de Bogotá ofertados por las universidades: Corporación Universitaria Minuto de Dios Virtual y a Distancia, Universidad Jorge Tadeo Lozano, Universidad Santo Tomás de Aquino, Pontificia Universidad Javeriana y Universidad Central, bajo la premisa de realizar un comparativo de rutas académicas e identificar elementos constitutivos de la formación de este perfil ocupacional a partir de seis categorías establecidas: cursos teóricos área de producción, cursos prácticos área de producción, cursos que tienen la comunicación como eje, cursos que tienen la investigación como eje, cursos que complementan el perfil del graduado de la universidad y cursos que complementan la labor del profesional. A continuación, se presenta el perfil ocupacional de los profesionales formados en los cinco programas objeto de este estudio: 
Tabla 1. Perfiles ocupacionales comunicación social IES Bogotá - Colombia.

\begin{tabular}{|c|c|c|}
\hline Universidad & Programa & Perfil ocupacional \\
\hline $\begin{array}{c}\text { Corporación Universitaria } \\
\text { Minuto de Dios Virtual y a } \\
\text { Distancia. }\end{array}$ & $\begin{array}{c}\text { Comunicación social, modalidad } \\
\text { virtual y a distancia. }\end{array}$ & $\begin{array}{l}\text { Creador y gestor de contenidos en medios tradicionales y } \\
\text { digitales. } \\
\text { Estratega en comunicación. } \\
\text { Gestor e investigador social. } \\
\text { Gestor de procesos culturales y organizaciones sociales. }\end{array}$ \\
\hline $\begin{array}{l}\text { Universidad Jorge Tadeo } \\
\text { Lozano. }\end{array}$ & Comunicación social y periodismo. & $\begin{array}{l}\text { Estratega en comunicación. } \\
\text { Realizador audiovisual y multimedia. } \\
\text { Periodista. } \\
\text { Investigador. } \\
\text { Gestor organizacional. } \\
\text { Consultor. }\end{array}$ \\
\hline $\begin{array}{c}\text { Universidad Santo Tomás de } \\
\text { Aquino. }\end{array}$ & Comunicación social. & $\begin{array}{l}\text { Investigador. } \\
\text { Productor. } \\
\text { Planeador, gestor y evaluador de proyectos de comunicación. } \\
\text { Mediador. }\end{array}$ \\
\hline $\begin{array}{l}\text { Pontificia Universidad Jave- } \\
\text { riana. }\end{array}$ & Comunicación social. & $\begin{array}{l}\text { Gestor de proyectos en todos los campos. } \\
\text { Realizador. } \\
\text { Periodista. } \\
\text { Gestor organizacional. }\end{array}$ \\
\hline Universidad Central. & Comunicación social. & $\begin{array}{l}\text { Investigador. } \\
\text { Productor. } \\
\text { Mediador. }\end{array}$ \\
\hline
\end{tabular}

Fuente. Perfiles ocupacionales, comunicación social IES Bogotá - Colombia (Martínez, 2018).

A partir de los perfiles ocupacionales definidos por las instituciones seleccionadas, se puede indicar que únicamente el programa de comunicación social, modalidad virtual y distancia de la Corporación Universitaria Minuto de Dios Virtual y a Distancia, contempla el perfil de creador y gestor de contenidos en medios tradicionales y digitales; los otros pro- gramas de forma implícita se acercan al perfil a través de perfiles asociados como estratega en comunicación, gestor de comunicación organizacional, mediador, productor o realizador. Descritos los perfiles ocupacionales de los programas académicos en estudio se presentan los componentes curriculares de cada uno de ellos:

Tabla 2. Componentes curriculares por tipo de curso comunicación social UVD.

\begin{tabular}{|c|c|c|c|c|}
\hline Universidad & Programa & Tipología de curso & $\begin{array}{l}\text { Número de } \\
\text { créditos por } \\
\text { area }\end{array}$ & $\begin{array}{l}\text { Número } \\
\text { de créditos } \\
\text { totales }\end{array}$ \\
\hline \multirow{7}{*}{$\begin{array}{c}\text { Corporación } \\
\text { Universitaria } \\
\text { Minuto de } \\
\text { Dios Virtual y a } \\
\text { Distancia. }\end{array}$} & \multirow{7}{*}{$\begin{array}{l}\text { Comunicación } \\
\text { social modalidad } \\
\text { virtual y a } \\
\text { distancia. }\end{array}$} & Cursos teóricos sobre la profesión & 9 & \multirow{7}{*}{144} \\
\hline & & Cursos prácticos sobre la profesión & 23 & \\
\hline & & Cursos que tienen la comunicación como eje & 34 & \\
\hline & & Cursos que tienen la investigación como eje & 15 & \\
\hline & & $\begin{array}{l}\text { Cursos que complementan el perfil del graduado de la } \\
\text { universidad }\end{array}$ & 21 & \\
\hline & & Cursos que complementan la labor del profesional & 33 & \\
\hline & & Electiva CPC & 9 & \\
\hline
\end{tabular}

Fuente. Componentes curriculares por tipo de curso comunicación social UVD (Cabrera, 2018). 
El programa de comunicación social de la Corporación Universitaria Minuto de Dios Virtual y a Distancia, cuenta con 144 créditos distribuidos en 10 períodos académicos. A nivel de ruta académica cuenta con 6 asignaturas específicas para la generación de un perfil de gestor de contenido o comunity manager: Di- seño y diagramación (3 créditos), Producción transmedia (3 créditos), Producción Web (3 créditos), Laboratorio de medios (2 créditos), Comunicación y participación en medios digitales (2 créditos) y Plan de medios digitales (2 créditos).

Tabla 3. Componentes curriculares por tipo de curso comunicación social y periodismo UJTL.

\begin{tabular}{|c|c|c|c|c|}
\hline Universidad & Programa & Tipología de curso & $\begin{array}{l}\text { Número de } \\
\text { créditos por } \\
\text { area }\end{array}$ & $\begin{array}{l}\text { Número } \\
\text { de créditos } \\
\text { totales }\end{array}$ \\
\hline \multirow{7}{*}{$\begin{array}{c}\text { Universidad } \\
\text { Jorge Tadeo } \\
\text { Lozano. }\end{array}$} & \multirow{7}{*}{$\begin{array}{c}\text { Comunicación } \\
\text { social y } \\
\text { periodismo. }\end{array}$} & Cursos teóricos sobre la profesión & 7 & \multirow{7}{*}{136} \\
\hline & & Cursos prácticos sobre la profesión & 21 & \\
\hline & & Cursos que tienen la comunicación como eje & 48 & \\
\hline & & Cursos que tienen la investigación como eje & 10 & \\
\hline & & $\begin{array}{l}\text { Cursos que complementan el perfil del graduado de la } \\
\text { universidad }\end{array}$ & 11 & \\
\hline & & Cursos que complementan la labor del profesional & 27 & \\
\hline & & Electivas & 12 & \\
\hline
\end{tabular}

Fuente. Componentes curriculares por tipo de curso comunicación social y periodismo UJTL (Cabrera, 2018).

El programa de comunicación social y periodismo de la Universidad Jorge Tadeo Lozano se condensa en 136 créditos, distribuidos en 8 semestres académicos. Integra asignaturas relacionadas a la generación de competencias TIC desde el núcleo de Fundamentación espe- cífica así: Comunicación y nuevas tecnologías (3 Créditos), Convergencia I (4 Créditos), Estrategias de comunicación digital (2 Créditos), Convergencia II (4 Créditos) y Laboratorio de medios (3 Créditos).

Tabla 4. Componentes curriculares por tipo de curso comunicación social Universidad Santo Tomás de Aquino.

\begin{tabular}{|c|c|c|c|c|}
\hline Universidad & Programa & Tipología de curso & $\begin{array}{l}\text { Número de } \\
\text { créditos por } \\
\text { area }\end{array}$ & $\begin{array}{l}\text { Número } \\
\text { de créditos } \\
\text { totales }\end{array}$ \\
\hline \multirow{7}{*}{$\begin{array}{c}\text { Universidad } \\
\text { Santo Tomás de } \\
\text { Aquino. }\end{array}$} & \multirow{7}{*}{$\begin{array}{l}\text { Comunicación } \\
\text { social. }\end{array}$} & Cursos teóricos sobre la profesión & 10 & \multirow{7}{*}{156} \\
\hline & & Cursos prácticos sobre la profesión & 14 & \\
\hline & & Cursos que tienen la comunicación como eje & 38 & \\
\hline & & Cursos que tienen la investigación como eje & 23 & \\
\hline & & $\begin{array}{l}\text { Cursos que complementan el perfil del graduado de la } \\
\text { universidad }\end{array}$ & 42 & \\
\hline & & Cursos que complementan la labor del profesional & 23 & \\
\hline & & Electivas & 6 & \\
\hline
\end{tabular}

Fuente. componentes curriculares por tipo de curso comunicación, Universidad Santo Tomás de Aquino (Cabrera, 2018). 
El programa de comunicación social de la Universidad Santo Tomás de Aquino se articula en 156 créditos distribuidos en 8 períodos académicos. Este programa tiene un componente TIC reducido, el cual se encuentra representado en dos asignaturas en sexto semestre: Comunicación y producción multimedia (3 Créditos) y Teoría y producción transmedia (2
Créditos), lo anterior se encuentra en relación con el perfil propuesto desde cuatro ámbitos de la profesión que se desarrollan curricular mente en séptimo y octavo semestre: Comunicación en conflicto, Comunicación organizacional, Comunicación-educación y Periodismo público.

Tabla 5. Componentes curriculares por tipo de curso comunicación social Pontificia Universidad Javeriana.

\begin{tabular}{|c|c|c|c|c|}
\hline Universidad & Programa & Tipología de curso & $\begin{array}{l}\text { Número de } \\
\text { créditos por } \\
\text { area }\end{array}$ & $\begin{array}{c}\text { Número } \\
\text { de créditos } \\
\text { totales }\end{array}$ \\
\hline \multirow{7}{*}{$\begin{array}{l}\text { Pontificia } \\
\text { Universidad } \\
\text { Javeriana. }\end{array}$} & \multirow{7}{*}{$\begin{array}{l}\text { Comunicación } \\
\text { social. }\end{array}$} & Cursos teóricos sobre la profesión & 27 & \multirow{7}{*}{178} \\
\hline & & Cursos prácticos sobre la profesión & 13 & \\
\hline & & Cursos que tienen la comunicación como eje & 41 & \\
\hline & & Cursos que tienen la investigación como eje & 11 & \\
\hline & & $\begin{array}{l}\text { Cursos que complementan el perfil del graduado de la } \\
\text { universidad }\end{array}$ & 5 & \\
\hline & & Cursos que complementan la labor del profesional & 52 & \\
\hline & & Electivas & 30 & \\
\hline
\end{tabular}

Fuente. Componentes curriculares por tipo de curso comunicación, Pontificia Universidad Javeriana (Cabrera, 2018).

El programa de comunicación social de la Pontificia Universidad Javeriana, contempla 178 créditos cursando un solo énfasis, 216 créditos cursando doble énfasis o 213 créditos si se cursan los énfasis: organizacional y publicidad o - periodismo y radio, estos créditos se distribuyen en 10 semestres académicos, los cuales se relacionan con 5 ejes específicos: Eje de filosofía, Eje de comunicación, Eje de estéticas, Ejes de investigación, Ejes de investigación y Eje de ciencias sociales; y 6 campos profesionales: audiovisual, periodismo, organizacional, publicidad, editorial y radio. Entre las asignaturas relacionadas con la perfilación del gestor de contenidos o CM se relacionan las siguientes: Técnicas computacionales comunicativas (2 créditos) - Directo transmedia (2 Créditos), Multimedia para las organizaciones (3 créditos), Cultura digital (4 créditos), In- formática para la edición (2 créditos), Proyecto digital en línea ( 3 créditos), Proyecto editorial digital (3 créditos), Diseño y programación de juegos (2 créditos) y Animación digital (2 créditos). 
Tabla 6. Componentes curriculares por tipo de curso comunicación social, Universidad Central.

\begin{tabular}{|c|c|c|c|c|}
\hline Universidad & Programa & Tipología de curso & $\begin{array}{l}\text { Número de } \\
\text { créditos por } \\
\text { area }\end{array}$ & $\begin{array}{l}\text { Número } \\
\text { de créditos } \\
\text { totales }\end{array}$ \\
\hline \multirow{7}{*}{$\begin{array}{l}\text { Universidad } \\
\text { Central. }\end{array}$} & \multirow{7}{*}{$\begin{array}{l}\text { Comunicación } \\
\text { social. }\end{array}$} & Cursos teóricos sobre la profesión & 13 & \multirow{7}{*}{140} \\
\hline & & Cursos prácticos sobre la profesión & 16 & \\
\hline & & Cursos que tienen la comunicación como eje & 20 & \\
\hline & & Cursos que tienen la investigación como eje & 8 & \\
\hline & & $\begin{array}{l}\text { Cursos que complementan el perfil del graduado de la } \\
\text { universidad }\end{array}$ & 35 & \\
\hline & & Cursos que complementan la labor del profesional & 9 & \\
\hline & & Electivas & 30 & \\
\hline
\end{tabular}

Fuente. Componentes curriculares por tipo de curso comunicación, Universidad Central (Cabrera, 2018).

El programa de comunicación social de la Universidad Central establece 140 créditos distribuidos en 9 semestres académicos En esta propuesta curricular no se marca ningún énfasis profesional específico, sin embargo, se cuenta con tres líneas de profundización disciplinar: Narrativas e imágenes, Comunicación y poder, y Tecnologías de la información y la comunicación (TIC), que se abordan desde tres ciclos formativos: Ciclo de reflexión conceptual (No se identificaron asignaturas para la generación de un perfil de CM), Ciclo de producción estética, en el cual se identificaron dos asignaturas pertinentes para la generación de un CM: Innovación en medios y lenguajes (4 créditos) y Cibercultura y comunicación contemporánea (3 créditos), y finalmente, el Ciclo de diseño y experimentación donde se encontraron seis cursos que permiten profundizar en la generación de competencia para la gestión del perfil ocupacional en estudio: Diseño audiovisual y digital (4 créditos), Diseño estratégico de procesos y redes de comunicación (3 créditos), Géneros, formatos e interactividad (3 créditos), Sistemas de datos (3 créditos), Conocimiento, tecnología y cultura ( 3 créditos) y Proyecto de línea (4 créditos).
En términos generales debe indicarse que la formación en comunicación social en IES privadas en Bogotá - Colombia, oscila entre los 8 y 10 semestres académicos, esta formación puede tener o no un énfasis específico, en la actualidad se encuentran los siguientes énfasis: conflicto, organizaciones, educación, periodismo, publicidad/diseño, editorial, radio, narrativas/imágenes/estética, política/ filosofía, investigación/experimentación en ciencias sociales, y Tecnologías de la información y la comunicación (TIC). Las asignaturas mayormente relacionadas con la formación de un perfil hiperespecializado en gestión de contenidos web, tipo comunity manager se pueden acoplar por grupos según componente temático en una propuesta académica de Medialab así: 
Tabla 7. Propuesta formativa continua y extracurricular para la formación de comunity managers - Medialab.

\begin{tabular}{|c|c|c|c|c|}
\hline Workshops & Cursos & Experimentación & Entrenamiento & Espacios de Networking \\
\hline $\begin{array}{l}\text { *Cultura Digital. } \\
\text { *Animación Digital. } \\
\text { *Comunicación y Nuevas } \\
\text { Tecnologías. } \\
\text { *Multimedia para las } \\
\text { Organizaciones. } \\
\text { *Conocimiento, } \\
\text { Tecnología y Cultura. } \\
\text { *Cibercultura y } \\
\text { Comunicación } \\
\text { *Contemporánea. } \\
\text { *Comunicación y } \\
\text { Participación en } \\
\text { Medios Digitales. }\end{array}$ & $\begin{array}{l}\text { *Diseño y Diagramación. } \\
\text { *Diseño Audiovisual y } \\
\text { Digital. } \\
\text { *Diseño y Programación } \\
\text { de Juegos. } \\
\text { *Diseño Estratégico de } \\
\text { Procesos y Redes de } \\
\text { Comunicación. }\end{array}$ & $\begin{array}{l}\text { *Sistemas de Datos. } \\
\text { *Directo Transmedia. } \\
\text { *Informática para la } \\
\text { Edición. } \\
\text { *Innovación en Medios y } \\
\text { Lenguajes. } \\
\text { *Géneros, Formatos e } \\
\text { Interactividad. } \\
\text { *Técnicas } \\
\text { Computacionales } \\
\text { Comunicativas. }\end{array}$ & $\begin{array}{l}\text { *Convergencia I. } \\
\text { *Convergencia II. } \\
\text { *Laboratorio de Medios. } \\
\text { *Proyecto Editorial } \\
\text { Digital } \\
\text { *Proyecto de Línea/ } \\
\text { Proyecto Digital en } \\
\text { Línea. }\end{array}$ & $\begin{array}{l}\text { *Producción Web. } \\
\text { *Teoría y Producción } \\
\text { Transmedia. } \\
\text { *Producción } \\
\text { Transmedia. } \\
\text { *Plan de Medios } \\
\text { Digitales. } \\
\text { *Estrategias de } \\
\text { Comunicación Digital. } \\
\text { *Comunicación } \\
\text { y Producción } \\
\text { Multimedia. }\end{array}$ \\
\hline
\end{tabular}

Fuente. Propuesta formativa continua y extracurricular para la formación de comunity managers - Medialab (Martínez, 2018).

Medialab, es un proyecto del programa comunicación social modalidad virtual y a distancia de la Corporación Universitaria Minuto de Dios, que busca ser un espacio de convergencia para la práctica de los procesos relacionados con el área de producción mediática del programa, desde lo operativo se construye en un espacio incrustado en la plataforma de aulas virtuales de la institución (http://aulas. uniminuto.edu), que vincula simuladores o programas de acceso en línea gratuito de las diferentes temáticas relacionadas al área de producción web. El éxito del Medialab se asocia al respeto por la planeación estratégica de la institución y del programa, en

atención a las necesidades de la modalidad en la cual está proyectado. Los módulos en los cuales está divido el proyecto actualmente son: Publicaciones digitales, Producción sonora, Producción audiovisual, Fotografía digital y Producción Web y Multimedia.

Son resultados del Medialab, el fortalecimiento de las capacidades de los estudiantes del comunicación social modalidad virtual y a distancia de la Corporación Universitaria Minuto de Dios, a través de procesos prácticos en los diversos proyectos extracurriculares que apoyan el programa, es por ello que en primera instancia se ha desarrollado una articulación el macroproyecto de programa denominado "Sala de prensa", un espacio de formación extracurricular abierto a toda la comunidad académica de Uniminuto virtual y a distancia.

El Medialab se convierte de esta forma, en el vehículo que permite que el estudiante trascienda las barreras técnicas en su proceso de aprendizaje y se adapte y aprenda procesos de producción de contenido, para la generación de productos en diversos escenarios mediáticos. 


\section{Conclusiones}

No existe un perfil formativo estático o definido para el comunity manager, sino que se trata de una figura profesional en continua evolución, de ahí que la formación no esté predeterminada (Treviño, Barranquero \& Zusberro. 2013) con respecto a esto Fuentes (1983) indica que "Desde hace más de una década, la reflexión de las escuelas de comunicación sobre su propio quehacer han ido definiendo una problemática creciente en importancia: el currículum académico se ha convertido en un tema recurrente de investigación y discusión", frente a esta afirmación debe indicarse que el currículo puede ser entendido de varias maneras, por ejemplo, para Stenhouse (1985, p.194221) citado por Luna (2011) “Un currículo es una tentativa para comunicar los principios y rasgos esenciales de propósitos educativos, de tal forma que permanezca abierta a la discusión crítica y pueda ser trasladado a la práctica".

Un diseño curricular debe respetar la orientación de la institución, su modelo educativo, y los intereses del sector productivo que buscan en el graduado una herramienta para el desarrollo de procesos comerciales, es por ello que la construcción del currículo debe ser un proceso elaborado, con una fuerte base metodológica. "El concepto de diseño curricular se refiere a la estructuración y organización de una serie de elementos orientados a la solución de problemas detectados previamente, donde es preciso considerar el conjunto de fases o etapas que se debe integrar en el proceso conducente a la conformación de un proyecto o propuesta curricular particular (Díaz Barriga, 1993, citado por Luna, 2011).
En consecuencia, plantear un diseño o rediseño curricular articulado al perfil del nuevo comunicador social, atendiendo a la hiperespecialización que se espera del campo implica la organización social de los saberes y la identidad disciplinaria del campo además de la planeación formativo - académica como proyecto de convergencia ante la trasformación social.

Lo anterior, hace necesario una revisión conceptual y práctica del concepto "comunity manager", para ofrecer una visión global actualizada de su origen, evolución y perspectivas futuras. De otro lado, es importante continuar con la exploración correlacional de la oferta académica en comunicación social no sólo desde el sector privado, la visión sobre la educación pública en este campo es fundamental, a raíz de esto se pretende generar una segunda fase de la investigación que permita dicha observación y la elaboración de un perfil profesional integral y prospectivo de la figura del comunity manager, acorde con la evolución de la comunicación social como campo.

Finalmente, cabe señalar que la oferta actual de IES privadas en comunicación social en Bogotá - Colombia, permite la formación de un perfil ocupacional en ámbitos relacionados con la sociedad digital mediática, no obstante, la formación en pregrado en este campo no está generando perfiles hiperespecializados de la profesión, en consecuencia, para entender mejor el impacto generado por este proyecto, es necesario evaluar las implicaciones de la apuesta extracurricular que se configura en un Medialab, incluso desde aspectos formativos en educación continuada. 


\section{Referencias}

Abela J. A. (2000). Las técnicas de análisis de contenido: una revisión actualizada. Recuperado de: http://maa.centrodeestudiosandaluces.es/biblio/imagendoc/00000001_00000 500/00000123/00000123_090h0101.PDF

Asociación Española de Responsables de Comunidad y Profesionales Social Media (AERCO) y Territorio Creativo (2009). La Función del comunity manager. Como las empresas están organizándose para crear y hacer crecer sus comunidades. Recuperado de: http://www.territoriocreativo.es/ etc/2009/11/community-managerwhitepaper.html

Bauman, Z. (9 de enero 2016). Las redes sociales son una trampa. Babelia. El País. Recuperado de: https:// elpais.com/cultura/2015/12/30/babelia/1451504427_675885.html

Bernete García, F. (2007). Revista Mediaciones sociales, 3, 11-26.

Borau Boira, E., García García, F. \& Rodríguez Gómez, A. (2013). La diplomacia del presente: universo 2.0 al alcance de la mano. Comunicación y hombre: revista interdisciplinar de ciencias de la comunicación y humanidades, 9, 75-88. Recuperado de: http:// dialnet.unirioja.es/servlet/articulo?codigo $=4546163$

Carlón, M. \& Neto, F. (Comps.). (2012). La política de los internautas: nuevas formas de participación. Buenos Aires: La Crujía.
Cobos, T.L. (2011). Y surge el community manager. Razón y palabra, 75. Recuperado de: http://www.razony palabra.org.mx/N/ N75/varia_75/varia2parte/15_Cobos_V75. pdf

Del Prado, S. (2011). Los 3 perfiles del comunity manager. Puro Marketing. Recuperado de: http://www.puromarketing. com/42/10353/perfiles-community-manager.html

DeFleur, M. L. \& Ball-Rokeach, S. (1989). Theories of Mass Communication. New York: David McKay.

DeFleur, M. L. (1968). White Plains, New York: Longma.

Díaz Barriga, F. (1993). Aproximaciones metodológicas al diseño curricular hacia una propuesta integral. Recuperado de http:// postgrado.una.edu.ve/curricular/paginas/ diazaproxima.pdf

Durkheim É. (2001 [1895]). Las reglas del método sociológico. Madrid: Akal.

Fernández, C., Galguera, L. (2009). Teorías de la comunicación. México: McGraw-Hill.

Gallegos, M. (28 de marzo de 2014) ¿Cuál es la voz de tu marca? [Mensaje en un blog] Recuperado de: http://communitymanagerslatam.com/cual-es-la-voz-de-tu-marca/

Gutiérrez, A. (2013). Community manager. Nuevo campo de acción para el comunicador organizacional. Tesis de pregrado. Pontificia Universidad Javeriana, Bogotá. Recuperado de: https://repository.javeria- 
na.edu.co/bitstream/handle/10554/11196/

GutierrezJerezAndresFernando2013.pdf?sequence $=1$

Hernández Sampieri, R., Fernández-Collado, C. \& Baptista Lucio, P. (2014). Metodología de la investigación. México: McGraw-Hill.

Krippendorff, K. (1990). Metodología de análisis de contenidos, teoría y práctica. Barcelona: Paidós.

Lara Muñoz, E. (2011). Fundamentos de investigación, un enfoque por competencias. México: Alfaomega.

López Noguero, F. (2002). Análisis de contenidos como método de investigación. XXI. Revista de educación, 4, 167-179.

Matías, P. (1998). La ciudad Internet. Madrid: Bellaterra.

Merejo, A. (2009). El cibermundo como entresijo virtual. Eikasia, Revista de Filosofía, 24. Recuperado de: http://revistadefilosofia. com/24-08.pdf

Moreno Reyes, F. (2015). Las redes sociales en Internet como herramienta de estimación del voto. (Tesis Doctoral). Universidad Complutense de Madrid, Madrid, España.

O'Reilly, T. (2005b). Web 2.0: compact definition. Recuperado de: http://radar.oreilly. com/archives/2005/10/web_20_compact_ definition.html

Ortega Maldonado, A. \& Becerra Muñoz, E. (2014). Estudio de la formación específica del community manager en la oferta formati- va española. E.Publishing Inc. Recuperado de: http://www.razonypalabra.org.mx/N/ N75/varia_75/varia2parte/15_Cobos_V75. Pdf

Ortega Maldonado, A. (2016) La formación especializada del community manager en el ámbito del estado español. Tesis doctoral, Departamento de Comunicación Audiovisual y Publicidad II. Universidad Complutense de Madrid: Madrid. Recuperado de: https://eprints.ucm.es/38082/1/T37364. pdf

Peña, P. (2001). Conocimiento. El oro gris de las organizaciones. Madrid: Fundación Dintel.

Revista Dinero. (28 de sept., 2017). Ranking de las marcas más poderosas en las redes sociales 2017. Recuperado de: https://www. dinero.com/edicion-impresa/caratula/articulo/ranking-de-los-reyes-de-las-redessociales/250385

Rizo, M. (2014). El papel de las teorías de la comunicación en la construcción del campo académico de la comunicación. Reflexiones desde la historia, la epistemología y la pedagogía. Correspondencia \& Análisis, 4, 239258.

Rojas, P. (2011). Community management para dummies. Madrid: Wiley Publishing Inc.

Salgado Lévano, A. C. (2007). Investigación cualitativa: diseños, evaluación del rigor metodológico y retos. Liberabit, 13(13), 7178.

Stenhouse, L.(1997). Investigación y desarrollo del currículum. Madrid: Morata. 
Sotomayor García, G. (2006). La socialización en los espacios virtuales. Posibilidades y limitaciones. Education in The Knowledge Society, 7(1).

Treviño, P., Barranquero, A. \& Zusberro, N. (2013). Community managers: presente y futuro de un perfil profesional emergente en la dimensión 2.0. Su influencia en la rentabilidad reputacional online. AdComunica, 6, recuperado de: http://www.adcomunicarevista.com/ojs/index.php/adcomunica/article/view/128

Wiener, N. (1988). Cybernetics, or Control and Communication in the Animal and the Machine. Cambridge MA: The MIT Press.

$$
\text { (c) (i) (O) }
$$

